\title{
MODELLING AN INTERACTIVE ROAD SIGNS SYSTEM, USING PETRI NETS
}

\author{
Timothee Kombe $^{1,2}$, Pierre Ele ${ }^{1}$, Florence Offole ${ }^{1}$, Herman Olivier Miasse ${ }^{1}$ \\ ${ }^{1}$ Laboratory of Electronic, Electrical Engineering, Automation \\ and Telecommunication University of Douala, Cameroon \\ ${ }^{2}$ e-mail:tkombe@yahoo.fr
}

\begin{abstract}
This paper is a contribution to the problems of road insecurity in Africa. Due to non- respect of road sign and to the lack of signing, roads have become places of all dangers. It becomes imperative to establish an interaction between the authorities and the offending drivers. To reach this goal, we modelled an interactive road-vehicle-signage system, who locally informs the driver on the requirements of traffic signs. This model having interest only in the event of driving by bad weather or deterioration of panels, we are amending by inserting functions aimed to warn and punish the driver in the event of maintenance of an offense. Indeed, when the driver is about to commit a fault, firstly the system issues a warming (visual, audible or mechanical). Then, a message (SMS) is sent to the authorities. We include the concept of floating process engaged by devices other than the signage. We show that, with a few considerations, from the functional point of view, they are identical to the process engaged by the signage. Furthermore, in terms of performance, the model renewed warnings that occurred just before the end panel of prohibitions. It stores messages of offenses occurred without the network, then notifies them when a network is detected. We propose algorithms for incremental design and analysis of the model, whose processes are activated and / or are extinguished, according to the type of sign or tag encountered. We show by simulation and by linear algebra that, the model retains its properties of absence of blocking and boundedness during the evolution of the system, hence its validation.
\end{abstract}

Keywords: Petri nets, interactive road signs, process, incremental design and analysis, linear algebra, floating process

\section{Introduction}

Road accidents are real threat to the world. They affect every country and every strata of the society. The International Traffic Safety Data and Analysis Group (2012) showed that in 2010, 1.3 million people across the world died of road accident. Without appropriate measures, the increase of road traffic may kill about 2.4 million people by 2030 and more than 50 million injured according to the World Health Organization in 2009. Road insecurity should be considered as a goal when it comes to development, particularly the millennium goal aimed at reducing poverty in the world by 2020 (World Health Organization, 2009).

Several factors favour the occurrence of road accidents. The quality of road signs, the visibility of the latter and their interpretation by drivers. The heavy toll of road accidents is assigned in the first spring to the human factor, in front of the vehicle status and the road infrastructure (International Federation of Red Cross and Red Crescent Societies, 2007) and (Belval, 2015). The defaults of road signs have a considerable influence in the occurrence of accidents (Zogo Tsala et al., 2015). The measures taken by the public authorities being ineffective, it would be indicated to propose a suitable tool which acts continuously on the human component, in order to make our roads safer.

Several approaches are used to conduct research in the field of road safety. One of the most commonly approach is that of detailed survey on road accidents (Organization for Economic Cooperation and Development, 1988). The principle of this approach is the detailed analysis of accidents based on the collection of data aimed at facilitating the understanding of factual progress and the relationship of casualty for each case study. According to Brenac and Fleury (1999), the detailed surveys approach can produce significant result on the evaluation of new technique measures, the evaluation of hypothesis or the emergence of new hypothesis regarding the occurrence of accidents and the possibility of prevention, when based on a conceptual framework and research explicit objective. However, difficulties are related to the need of implementing a multidisciplinary expertise for a detailed analysis of cases and rely on data collection. Such approach used in France for detailed accidents surveys programs were implemented since 1980 (Brenac, 1997), following a first restricted program realized between 1965 and 1969. (Fanga et al., 2004) proposed a computational model used to develop a system for automatically detecting and recognizing road signs from sequences of traffic images. In (Young et al., 2014), the state of the art in the use of computer models to simulate the level of safety in the traffic system was done. The aim was to review computer models of driver and vehicle behaviour within a road context. It focused on stochastic numerical models of traffic behaviour and how reliable these are in estimating levels of safety on the traffic network. The interest of our paper is undeniable. It allows modelling the interaction road-traffic sign-vehicle for the integration of the driver's offenses. The fines imposed on offending drivers by authorities may appear to be an effective way that can improve road safety, especially in developing countries. 


\section{Modelling the Studied System}

The model is a representation of the reality in formalism; it is an abstraction of a real system that already or does not exist (Kombe, 2011). Many approaches adopted Petri nets as basic formalism for modelling dynamic systems (Bastide, 2000). Petri nets are renowned for their ability to respond to this request, especially for Discrete Events Systems (DES) (Cassandras and Lafortune, 2008) that make up the road, its signage and users. In fact, Petri nets are a tool that is both mathematical and graphical. As mathematical tool, it allows to analyze the properties of the system and to characterize its activities by a set of states equations (Chu, 1995). As a graphical tool, it is a bipartisan graph constitutes of places and transitions connected by arcs. Petri nets therefore seem to be one of the tools that can model, analyze and evaluate systems during their design and operation.

Other tools such as Stochastic Processes Algebra and Stochastic Automata Networks (Atif, 1993) are also used. These approaches do not have the concept of entities and flow (respectively tokens and arcs in Petri nets). However, they offer a compositional vision of subsystems (Fernandes, 1998). The Stochastic Automata Networks is a high-level formalism that allows modelling very large and complex Markov chain. They were built to take into account the size of the state space during the conception of a model. In this formalism, the behaviour of a component of a system is represented by a set of states which is an automaton (a Markov chain with a reasonable state space). Each automaton is described by a local matrix. The set of all automata thus formed is represented by a multidimensional Markov chain, whose states are those of the product space, with an underlying matrix obtained by applying the operators of the tensor algebra (or Kronecker) on local matrices of each automaton. Effective resolution techniques are then available for quantitative analysis. But, the use of independent components connected via synchronizations functions can produce a state space with many inaccessible states. To model complex and dynamic system, the used tool must operate without bug. In our work, the combination of Petri nets with linear algebra allowed to correctly modelling the studied system. We showed by simulation using the Toolkit for Interactive Network Analysis (TINA), that the model retains its properties of absence of blocking and boundedness during the evolution of the system

\subsection{Modelling the road-tag (sign panel)-vehicles system using the Petri Nets}

The tags $\mathrm{T}$ broadcast locally the prescription of the sign panel that it dematerializes while $\mathrm{P}$, the embedded system in the car picks up this signal and informs the driver. In the Figure 1, the places P1, P2, P3 and P4 represent the vehicle status while travelling in the road, respectively after having met the T4, T1, T2 and T3 fixed tags (sign panels) represented by the transitions of the same name. These places and transitions are described in Table 1. This sub-system constitutes a Graph of Events (GE), showing that, any place has exactly one input and one output transition.

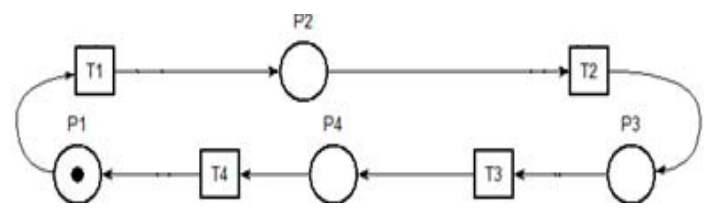

Figure 1. Sub-system: road-tags-vehicles

A GE being a network without conflict and view its properties, our model is thus deemed valid because it presents no risk of blockage or accumulation of resources. However, from the point of view of the exploitation of the prescriptions of the panels, it falls in the case of figure where the driver reads directly the prescriptions on the road signs. This is only of interest when the weather conditions or the wear makes difficult the reading of panel (rain, night, panel damaged). It would be therefore indicated that the system compares the requirements of the panels to the pace of the vehicle and, in the case of gap, warn the driver so that he corrects and avoids an accident or the Administration's sanctions.

\subsection{Application of the model to the prevention and the sanction of breaches of the Highway Code}

We hope that the firing of a transition engaged the execution of the process corresponding to the management of prescription of the sign panel. We denote by $\mathrm{T} 1$ the panel marking the beginning of a prescription and T4 the one marking its end. The same is true for T2 respectively T3. The Figure 2 describes the model while the Table 1 describes the places and nodes of the subsystems.

When the vehicle is within the field of the $\mathrm{T} 1 \mathrm{or} \mathrm{T} 2 \mathrm{tag}$, the corresponding panel management process begins. These processes being identical, we describe the process 1 related to $\mathrm{T} 1$ panel. In the 
place P5, the panel parameters are updated; they are regenerated by the firing of T5 and finally consumed by the firing of the end panel. In the place P7 who plays the role of memory, a mark shows that the process is underway and that the panel parameters are valid. The absence of marks puts end to the consideration of Panel parameters. This marking is consumed by a firing of the transition of the end panel. In T5, the calculator compares the measurements from the sensors to the Panel parameters. In case of non-compliance with the prescriptions, a firing of T5 puts a mark in P9. The firing of T5 also puts a mark in P5, so that new violations are taken into account until the end panel is meeting. In P9, the marks are accumulated, making fireable T11. At each arrival of a marking, the driver is warned by a "beep", a sound, or a light indicator

The firing of T11 puts a token in P16, making fireable T14 while for T13, there must also be that the process is underway (token or marks in P7 or P8 show that the corresponding process is underway) and that the sensors that have reported the infraction are still positioned (token or marks in P13 and P14 indicates that the corresponding sensor are positioned). Two cases can occur:

- T13 is not fireable. This means that the driver has corrected the infraction after that the warning was given, or the process was completed. Therefore, the conflict is effective when T13 and T14 are fireable at the same time. In that case, the level of priority must be defined in the software. The firing of T14 consumes the token in P16. The SMS of withdrawal of penalty points system of the driving license is prepared and stored in P18, making T16 fireable. A firing of T16 loads the place P19 which stores the SMS pending the availability of the network. The firing of T16 returns the mark in the place P15, making thus available the SMS processing subroutine.

- T13 is fireable. This means that the driver did not correct the infraction despite warning. The firing of T13 loads a token in P17 and the following is identical to the previous case, the only difference being here that, two SMS are generated: one for the withdrawal of the penalty points system of the driving license and the other for the fine. A firing of T15 loads the two SMS in T19 and returns the token in the place P15, making thus available the SMS processing subroutine.

While in its way, the vehicle enters in the field of the tag T4, which marks the end of T1 panel, the corresponding panel management process begins: In PT1, the panel parameters are updated; if the T1 panel process is in progress, the transition TT1 is fired, thus ending the process. The P7 memory is reset. Otherwise, we are in the case where the car meets an end panel before the one of the beginning, the firing of Tt1 consumes the mark in PT1. This is a way of shedding (loop exit).

\subsection{The floating process}

We are talking here about the process engaged by devices other than the signs. These devices represent particularly the sensors, the misinterpretations circulation tags and the tags embedded in the vehicles. The floating processes are proposed here to deal with the misinterpretations circulation, the third position and the changes in direction of the vehicle. The floating process is materialized (Fig. 3) by the ellipse in red in the model. It is identical from the point of view of operation with those of panels in considering the states of Ft 10 sensor as start panel and Ft8 as end panel. See in blue, a process engaged by a tag panel of signalling and in green the states of a sensor.

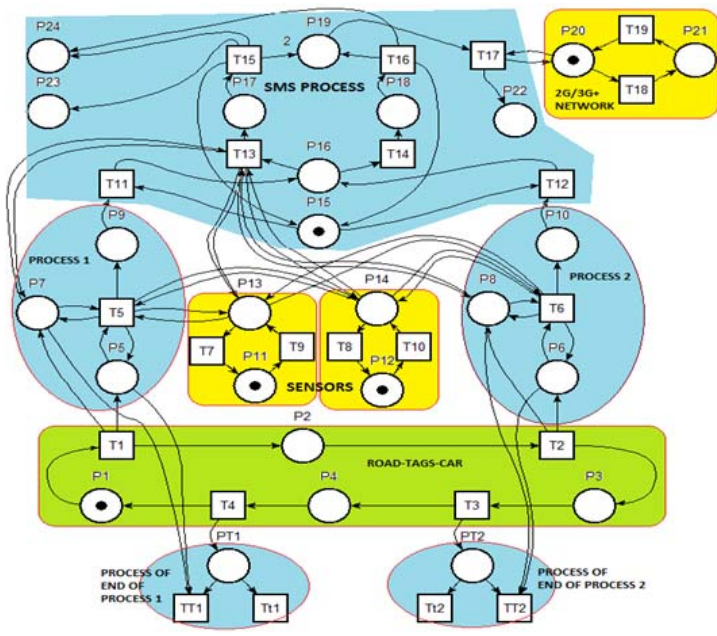

Figure 2. Model of interactive signaling system integrating the prevention and punishment of breaches

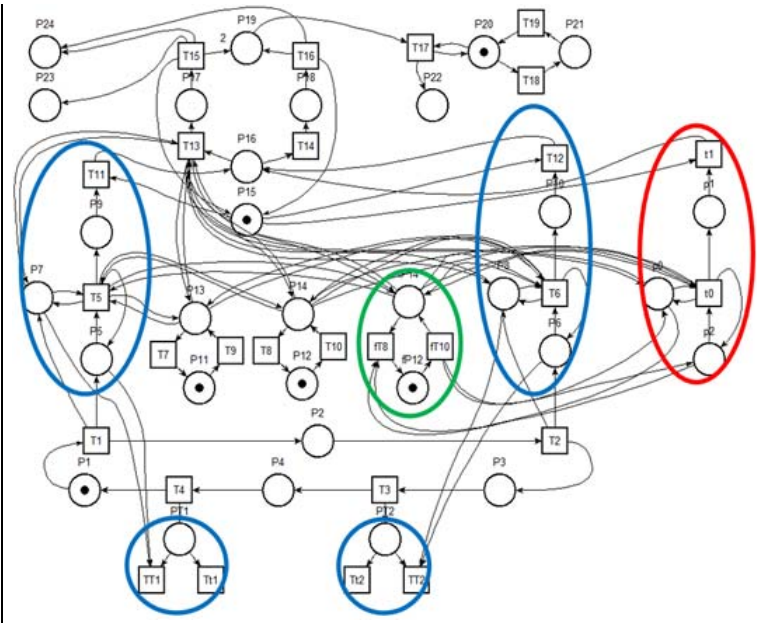

Figure 3. Model with all processes 


\section{Validation of the Model by the Simulation}

The screen capture of simulation (Fig. 4) fact state of 21 SMS transmitted (P22), 09 waiting for the presence of the GSM network (P19). These SMS are divided into 06 fines (P23) and 24 warnings (P24); in addition, breaches are pending the shared resource in (P15) for processing, either 10 for the process 1 (P9) and 12 for the process 2 (P10).

The accumulation of 08 tokens in P5 and 10 in P6 is only apparent and means that, for a given prescription, new signs of beginning have successively been encountered by the vehicle, before the end panel. This shows the limits of the simulation. In fact, TT1 and TT2 are sometimes checked instead of TT1 and TT2 which should stop the process thereto. This is corrected by treating the process of end panel to the transition T4 (Fig. 5).

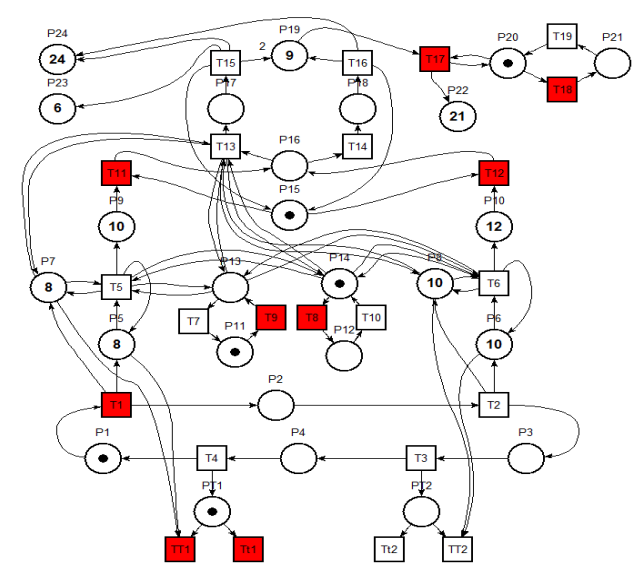

Figure 4. Screen capture at a given moment

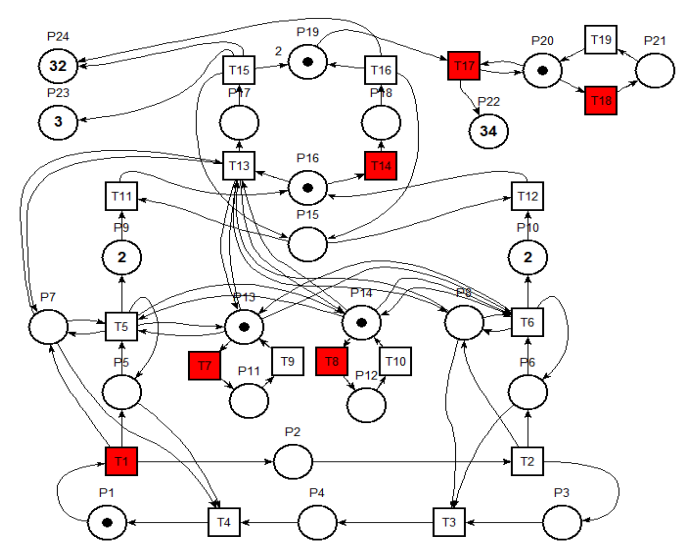

Figure 5. Tokens or markings "yellow"

The cycles of the processes 1 and 2 are completed, while the buffers of warnings P9 and P10 contain respectively 2 and 2 tokens representing warnings for breaches (Fig. 5). This may result from the fact that the vehicle has passed the end panel, while another process (manager of messages) was mobilizing the shared resource or that the violation of parameters occurred during the meeting of the end panel.

Although, the sensors are in breaches position, these are no longer taken into account. The tokens are therefore treated as warning one after the other, until the depletion of stocks in P9 and P10. In effect, as long as these places contain tokens and that the resource in P15 is available; the transitions T11 and T12 are enabled. However, as soon as a new process is engaged while there are still tokens in stock, any new breaches is regarded as refusal to comply with the reported warning and processed as penalty. Everything is happening as the yellow cards in soccer who will keep on several meetings, where we introduce the appellation of yellow markings or tokens. So, we outline of the property or the performance of conservation of warnings (mistakes) during the extinction of a process.

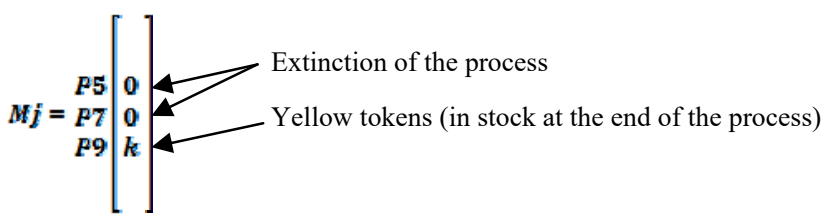

In the absence of the network GSM/ 3G+, the messages are stored, and then fully transmitted of the presence of the network, even if the vehicle is parked. The notification of infringements to the Administrations is independent of the place and time where they have been committed. However, these data are contained in the transmitted message.

No apparent lock not being observed; we validate the model by the simulation. However, the simulation is not an exhaustive analysis method. So, we combine it with the linear algebra analysis method. 
Table 1. Description of sub-systems

\begin{tabular}{|c|c|c|}
\hline Sub systems & $\begin{array}{l}\text { Places/ } \\
\text { Transitions }\end{array}$ & Description \\
\hline \multirow{8}{*}{$\begin{array}{l}\text { Road-Tags- } \\
\text { vehicle }\end{array}$} & $\mathrm{P} 1$ & State of the vehicle after the meet of the panel giving end to the panel T1 \\
\hline & $\mathrm{P} 2$ & State of the vehicle executing the prescriptions of the panel T1 \\
\hline & P3 & State of the vehicle executing the prescriptions of the panel T2 \\
\hline & $\mathrm{P} 4$ & State of the vehicle after the meet of the panel giving end to the panel T2 \\
\hline & $\mathrm{T} 1$ & A tag broadcasting the prescriptions of the sign panel 1 \\
\hline & $\mathrm{T} 2$ & A tag broadcasting the prescriptions of the sign panel 2 \\
\hline & $\mathrm{T} 3$ & A tag putting end to the prescriptions of the sign panel 2 \\
\hline & $\mathrm{T} 4$ & A tag putting end to the prescriptions of the sign panel 1 \\
\hline \multirow{4}{*}{$\begin{array}{l}\text { Processes } \\
1 \text { and } 2\end{array}$} & P5, P6 & Update Parameters to be controlled transmitted by panel \\
\hline & P7, P8 & Flag of validity of parameters \\
\hline & P9, P10 & Processing of detected breaches \\
\hline & T5, T6 & Comparison of sensors' parameters to those prescribed by the panel \\
\hline \multirow{3}{*}{$\begin{array}{c}\text { End of } \\
\text { processes } \\
1 \text { and } 2\end{array}$} & PT1, PT2 & Update Parameters not to be controlled again \\
\hline & TT1, TT2 & Extinction of the process of control of prescriptions of the respective panels \\
\hline & $\mathrm{Tt} 1, \mathrm{Tt} 2$ & $\begin{array}{l}\text { Cancellation of the parameters passed by the tags } \mathrm{T} 3 \text { and } \mathrm{T} 4 \text { in case the respective panels } \\
\mathrm{T} 2 \text { and } \mathrm{T} 1 \text { have previously not been encountered by the vehicle }\end{array}$ \\
\hline \multirow{13}{*}{$\begin{array}{l}\text { Edition of } \\
\text { SMS }\end{array}$} & $\mathrm{P} 15$ & Sub-program of SMS editing shared by all processes \\
\hline & P16 & Loading breaches stored in P9 and P10 \\
\hline & P17 & SMS edition for fine and withdrawal of the points of the driving license \\
\hline & P18 & SMS edition for withdrawal of the points of the driving license \\
\hline & P19 & SMS storage pending the network for transmission \\
\hline & $\mathrm{P} 22$ & Transmitted SMS counter \\
\hline & $\mathrm{P} 23$ & Counter of fines SMS \\
\hline & $\mathrm{P} 24$ & Counter of SMS for withdrawal of points of the driving license \\
\hline & $\mathrm{T} 11, \mathrm{~T} 12$ & Loading of the breaches when the sub-program of SMS is free \\
\hline & $\mathrm{T} 13$ & Detection of keeping the breaches despite the warning \\
\hline & $\mathrm{T} 14$ & Detection of the respect of the warning \\
\hline & $\mathrm{T} 15, \mathrm{~T} 16$ & Loading written SMS in memory and liberation of the sub-program \\
\hline & $\mathrm{T} 17$ & Detection of the network for the transmission of SMS \\
\hline \multirow{4}{*}{$\begin{array}{l}2 \mathrm{G} / 3 \mathrm{G} \\
\text { Network }\end{array}$} & $\mathrm{P} 20$ & Presence of network indicator \\
\hline & $\mathrm{P} 21$ & Absence of network indicator \\
\hline & T18 & Change from the present state to absent state \\
\hline & T19 & Change from the absent state to present state \\
\hline \multirow{4}{*}{ Sensors } & $\mathrm{P} 11, \mathrm{P} 12$ & Indicator of normal value \\
\hline & P13, P14 & Threshold overflow indicator \\
\hline & $\mathrm{T} 7, \mathrm{~T} 8$ & Switching the overflow state to the normal state \\
\hline & T9, T10 & Switching the normal state to the overflow state \\
\hline
\end{tabular}




\section{Validation of the Model by the Linear Algebra}

The analysis by linear algebra is based on the fundamental equation of the crossing; it allows exploring the properties of a network, including the terminal character, the vivacity and the absence of blocking, independently of an initial marking. Because of this, there will be talk of structural properties of the network. As well, we will be told of a network, that it is structurally terminal if it is terminal for any finished initial marking. Similarly, if for any initial marking, the network is alive, we will say that it is structurally living. This analysis is all the more relevant that we cannot determine in advance in what initial conditions the vehicle will get accost the road. The model should be valid regardless of the state of the system at a moment considered as initial (an initial marking).

\subsection{Characteristic Equation of crossing}

The characteristic equation of firing is given by the expression $M=M o+C V \varsigma$, where $M$ is the marking at a given moment obtained from the initial marking $M o$ if one fires the transition $\sigma$, assuming that $\sigma$ being traversable; $V \sigma$ Corresponds to several sequences of firing some of which are not penetrable; $C$ is the impact matrix (Table 2).

\subsection{The analysis}

The analysis is based on the following properties:

1. A living network is without blocking (Leclercq, 2012) and (Bennis, 2014);

2. If there is $\boldsymbol{f}>\mathbf{0}$ and $i$ such as $f^{t} \leq 0$ and $f^{t} C_{i}<0$ then the network is living for no initial marking and the transition $i$ is responsible. In addition, the network is structurally terminal (Chu, 1995) and (Hamdi Hocine, 2003).

So we are looking for our model, the possible transitions that could make the network non-living and therefore block the system. The resolution of this inequation gives the following relationships: $\mathrm{a}=\mathrm{b}=$ $\mathrm{d}=\mathrm{c} ; \mathrm{v}=\mathrm{w}=\mathrm{i}=\mathrm{j}=\mathrm{s} ; \mathrm{e}=-\mathrm{g} ; \mathrm{f}=-\mathrm{h} ; \mathrm{k}=\mathrm{m} ; \mathrm{l}=\mathrm{n} ; \mathrm{p}=\mathrm{q}=\mathrm{r}=\mathrm{o} ; \mathrm{u}=\mathrm{t}$.

Considering $\boldsymbol{f}>0$, we have $\boldsymbol{f}^{\boldsymbol{t}} \leq \boldsymbol{0}$ and $\boldsymbol{f}^{t} \boldsymbol{C}_{\boldsymbol{i}}<\boldsymbol{0}$ for $\mathrm{i}=$ TT1 and $i=T T 2$

The transitions TT1 and TT2 are therefore the only responsible for the fact that the network will not be living. This is not blocking the entire network, but the processes using these transitions; this corresponds well to the execution of the signals of end tags of panels that put an end to the process of panel management. Indeed, during the period of the lock, the requirements controlled by the process are no longer taken into account (speed limits, prohibition of exceed etc.).

\subsection{Generalization: Algorithms for incremental design}

We have shown that the Petri nets model of our system is without blocking. In fact, the blockages encountered are those desired and which mark the end of the process. In addition, we have shown that the places connected to the transitions responsible for the "controlled Lock" are bounded. However, we are in the incremental design phase where the network evolves with the occurrence of events. The meeting of new panels enables or disables processes, which is reflected in our model by adding or removing the places, transitions or sub-networks. Therefore, it would be indicated to know if during its evolution, the model retains its properties of absence of blocking and its boundedness.

\subsection{Deletion of places, transitions, and sub-networks}

When the vehicle handling two pairs of panels met with one of the end signs, the process related to this signalling panel is extinguished (beginning and end). It follows the removal of places, transitions, and sub-networks constituting these processes (Fig. 6). Only, a pair of road signs (two processes) remains in course of treatment in the vehicle. In Figure 6, we mark the extinguished process with red circles.

Because of this, the place P2 and transition T4 are directly connected by the arc P $\rightarrow$ T4. This implies that the resource P15 is no longer shared and is devoted exclusively to the current process. The resulting impact matrix returns to delete in the previous (Table 2), the columns corresponding to the transitions T2, TT2, T3, T6, T12, Tt2 and the lines relating to the placed P3, P4, P6, P8, P10, P12. In Table 3, which follows, the rows and columns removed are highlighted.

Furthermore, as a result of arc P2 $\rightarrow \mathrm{T} 4$, we replace $\mathbf{0}$ by $\mathbf{- 1}$ in the cell (P2, T4) of matrix. This illustrates that, the firing of the transition T4 removes a token from place P2. 


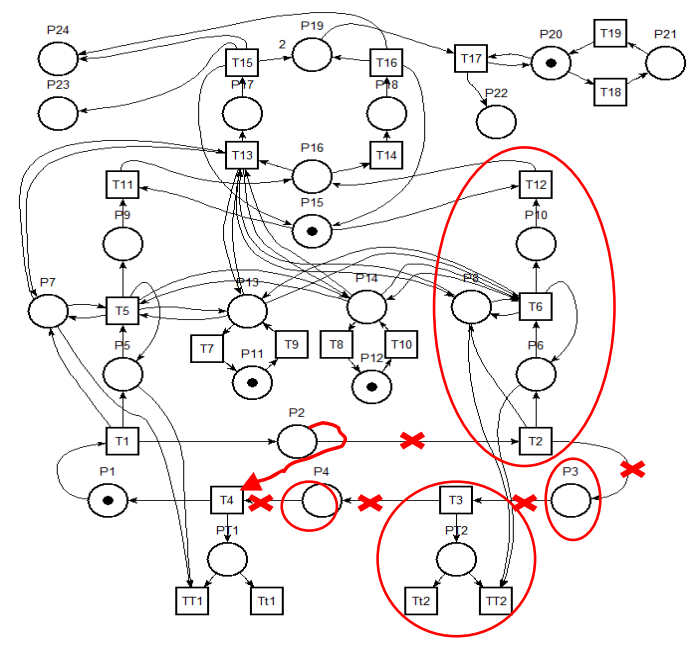

Figure 6. Extinction of a panel: Deletion of places, transitions, and sub-networks

The analysis of the model in view of the research of the possible transitions that could block the system derives from the properties 1 and 2 of the paragraph 4.2:

The resolution of the in equation $\boldsymbol{f}^{t} \mathrm{C} \leq 0$ gives the following relationships: $\mathrm{q}=\mathrm{e}=\mathrm{n}=0 ; \mathrm{a}=\mathrm{b} ; \mathrm{f}$ $=\mathrm{h} ; \mathrm{g}=\mathrm{i} ; \mathrm{k}=\mathrm{l}=\mathrm{m}=\mathrm{j} ; \mathrm{o}=\mathrm{p}$; and $-\mathrm{c}-\mathrm{d}-\mathrm{q} \leq 0$ is true because $\mathrm{f}>0$. We have therefore $\boldsymbol{f}^{\boldsymbol{t}} \leq \boldsymbol{0}$ and $\boldsymbol{f}^{t} \boldsymbol{C}_{\boldsymbol{i}}<\boldsymbol{0}$ for $i=T T 1$.

The transition TT1 is the only one responsible for the fact that the network will not be living. It corresponds well to the end of the process sought by the prescription end tag. In addition, P5 and P7, the space attached to this transition, are 1- bounded. In view of the foregoing, we propose the algorithm below for the conservation of the absence of blocking and the boundedness of our model, following the withdrawal of the places and transitions on the sub-system GE:

1. Define $\mathrm{P}^{*}$ the "downstream space" (downstream of the deletion) corresponding to the space connected to first transition to delete in the Graph of Events; and * $\boldsymbol{T}$ the «upstream transition» corresponding to the transition connected to the last space to delete in the Graph of Events, in the sense of evolution of the system;

2. Connect the downstream space to the upstream transition $\left(\operatorname{arc} P^{*} \rightarrow * T\right)$;

3. Delete in the Impact matrix, the rows and columns relating respectively to the deleted spaces and transitions;

4. Set to -1 the cell $\left(\boldsymbol{P}^{*}, * \boldsymbol{T}\right)$ and keep the rest of the array without change.

Table 2. Impact Matrix (C) of the model

\begin{tabular}{|c|c|c|c|c|c|c|c|c|c|c|c|c|c|c|c|c|c|c|c|c|c|c|c|}
\hline & $\mathrm{T} 1$ & TT1 & $\mathrm{Tt} 1$ & T2 & TT2 & $\mathrm{Tt} 2$ & $\mathrm{~T} 3$ & T4 & T5 & T6 & $\mathrm{T} 7$ & T8 & T9 & $\mathrm{T} 10$ & T11 & $\mathrm{T} 12$ & $\mathrm{~T} 13$ & T14 & T15 & T16 & T17 & T18 & $\mathrm{T} 19$ \\
\hline $\mathrm{P} 1$ & -1 & 0 & 0 & 0 & 0 & 0 & 0 & 1 & 0 & 0 & 0 & 0 & 0 & 0 & 0 & 0 & 0 & 0 & 0 & 0 & 0 & 0 & 0 \\
\hline P2 & 1 & 0 & 0 & -1 & 0 & 0 & 0 & 0 & 0 & 0 & 0 & 0 & 0 & 0 & 0 & 0 & 0 & 0 & 0 & 0 & 0 & 0 & 0 \\
\hline P3 & 0 & 0 & 0 & 1 & 0 & 0 & -1 & 0 & 0 & 0 & 0 & 0 & 0 & 0 & 0 & 0 & 0 & 0 & 0 & 0 & 0 & 0 & 0 \\
\hline P4 & 0 & 0 & 0 & 0 & 0 & 0 & 1 & -1 & 0 & 0 & 0 & 0 & 0 & 0 & 0 & 0 & 0 & 0 & 0 & 0 & 0 & 0 & 0 \\
\hline P5 & 1 & -1 & 0 & 0 & 0 & 0 & 0 & 0 & 0 & 0 & 0 & 0 & 0 & 0 & 0 & 0 & 0 & 0 & 0 & 0 & 0 & 0 & 0 \\
\hline P6 & 0 & 0 & 0 & 1 & -1 & 0 & 0 & 0 & 0 & 0 & 0 & 0 & 0 & 0 & 0 & 0 & 0 & 0 & 0 & 0 & 0 & 0 & 0 \\
\hline P7 & 1 & -1 & 0 & 0 & 0 & 0 & 0 & 0 & 0 & 0 & 0 & 0 & 0 & 0 & 0 & 0 & 0 & 0 & 0 & 0 & 0 & 0 & 0 \\
\hline P8 & 0 & 0 & 0 & 1 & -1 & 0 & 0 & 0 & 0 & 0 & 0 & 0 & 0 & 0 & 0 & 0 & 0 & 0 & 0 & 0 & 0 & 0 & 0 \\
\hline P9 & 0 & 0 & 0 & 0 & 0 & 0 & 0 & 0 & 1 & 0 & 0 & 0 & 0 & 0 & -1 & 0 & 0 & 0 & 0 & 0 & 0 & 0 & 0 \\
\hline P10 & 0 & 0 & 0 & 0 & 0 & 0 & 0 & 0 & 0 & 1 & 0 & 0 & 0 & 0 & 0 & -1 & 0 & 0 & 0 & 0 & 0 & 0 & 0 \\
\hline P11 & 0 & 0 & 0 & 0 & 0 & 0 & 0 & 0 & 0 & 0 & 1 & 0 & -1 & 0 & 0 & 0 & 0 & 0 & 0 & 0 & 0 & 0 & 0 \\
\hline $\mathrm{P} 12$ & 0 & 0 & 0 & 0 & 0 & 0 & 0 & 0 & 0 & 0 & 0 & 1 & 0 & -1 & 0 & 0 & 0 & 0 & 0 & 0 & 0 & 0 & 0 \\
\hline $\mathrm{P} 13$ & 0 & 0 & 0 & 0 & 0 & 0 & 0 & 0 & 0 & 0 & -1 & 0 & 1 & 0 & 0 & 0 & 0 & 0 & 0 & 0 & 0 & 0 & 0 \\
\hline P14 & 0 & 0 & 0 & 0 & 0 & 0 & 0 & 0 & 0 & 0 & 0 & -1 & 0 & 1 & 0 & 0 & 0 & 0 & 0 & 0 & 0 & 0 & 0 \\
\hline P15 & 0 & 0 & 0 & 0 & 0 & 0 & 0 & 0 & 0 & 0 & 0 & 0 & 0 & 0 & -1 & -1 & 0 & 0 & 1 & 1 & 0 & 0 & 0 \\
\hline P16 & 0 & 0 & 0 & 0 & 0 & 0 & 0 & 0 & 0 & 0 & 0 & 0 & 0 & 0 & 1 & 1 & -1 & -1 & 0 & 0 & 0 & 0 & 0 \\
\hline P17 & 0 & 0 & 0 & 0 & 0 & 0 & 0 & 0 & 0 & 0 & 0 & 0 & 0 & 0 & 0 & 0 & 1 & 0 & -1 & 0 & 0 & 0 & 0 \\
\hline P18 & 0 & 0 & 0 & 0 & 0 & 0 & 0 & 0 & 0 & 0 & 0 & 0 & 0 & 0 & 0 & 0 & 0 & 1 & 0 & -1 & 0 & 0 & 0 \\
\hline P19 & 0 & 0 & 0 & 0 & 0 & 0 & 0 & 0 & 0 & 0 & 0 & 0 & 0 & 0 & 0 & 0 & 0 & 0 & 2 & 1 & -1 & 0 & 0 \\
\hline P20 & 0 & 0 & 0 & 0 & 0 & 0 & 0 & 0 & 0 & 0 & 0 & 0 & 0 & 0 & 0 & 0 & 0 & 0 & 0 & 0 & 0 & -1 & 1 \\
\hline P21 & 0 & 0 & 0 & 0 & 0 & 0 & 0 & 0 & 0 & 0 & 0 & 0 & 0 & 0 & 0 & 0 & 0 & 0 & 0 & 0 & 0 & 1 & -1 \\
\hline PT1 & 0 & -1 & -1 & 0 & 0 & 0 & 0 & 1 & 0 & 0 & 0 & 0 & 0 & 0 & 0 & 0 & 0 & 0 & 0 & 0 & 0 & 0 & 0 \\
\hline PT2 & 0 & 0 & 0 & 0 & -1 & -1 & 1 & 0 & 0 & 0 & 0 & 0 & 0 & 0 & 0 & 0 & 0 & 0 & 0 & 0 & 0 & 0 & 0 \\
\hline
\end{tabular}


By applying this algorithm in our model, one gets well the Petri nets of Figure 6 and the impact matrix of the Table 3 as follows:

1. Set $\boldsymbol{P}^{*}=\mathrm{P} 2$ and ${ }^{*} \boldsymbol{T}=\mathrm{T} 4$;

2. Join P2 to T4 (Fig. 6);

3. Delete the rows and columns of the places and transitions to delete (Table 3);

4. Set to -1 the cell $(\mathrm{P} 2, \mathrm{~T} 4)$ and keep the rest of the array without change.

\subsection{Adding of places, transitions and sub-networks}

When the vehicle handling two pairs of panels met a third panel, the process related to this signalling panel is activated (beginning and end). It follows in our initial model (Fig. 2), the addition of places, transitions, and sub-networks constituting these processes. The result is the Petri nets of the Fig. 7.

The transformation has been to open the arc $\mathbf{P 3} \rightarrow \mathbf{T 3}$ and to insert: the transition Ti, the subsystem (the process of management of the new panel) and the places Pi thereto; the transition TTi, the sub-system (the process of Ti end panel) and the place PPI thereto. The process of managing the new panel is constituted of places PI1, Pi2, Pi3 and transitions Ti1, Ti2. The process of end panel Ti is constituted of the places PTI and transitions Tti1, TTi1. The red arc connects the inserted elements to the system. The result is the characteristic matrix represented in Table 4.

The analysis of the model in view of the research of the possible transitions that could block the system derives from the properties 1 and 2 of the paragraph $4.2: \boldsymbol{f}^{t} \boldsymbol{C} \leq \mathbf{0}$ is true for $\boldsymbol{- e}-\boldsymbol{g}-\boldsymbol{v}$, $-\boldsymbol{f}-\boldsymbol{h}-\boldsymbol{w}$, and $-\boldsymbol{z}-\boldsymbol{A}-\boldsymbol{C}$, whether $\boldsymbol{f}^{\boldsymbol{t}} \boldsymbol{C}_{\boldsymbol{i}}<0$ for $i=\boldsymbol{T T 1}$, TT2 and TTi1.

These transitions linked to processes of end panels are the only ones responsible for the fact that the network will not be living and, consequently, of the blocking of the signs management processes they control. In addition, the places P7, P5; P6, P8 and Pi1, Pi3 related respectively to these transitions are bounded.

The model of the system therefore retains its properties of absence of blocking (control blocking) and boundedness (localized) during its incremental evolution. Also, we propose below, the algorithm of model transformation for the purposes of inserting of new processes, while maintaining those properties:

1. Open the arc linking the place marking the current state of the vehicle to the next transition;

2. Define $\boldsymbol{P}^{*}$ the "downstream space» and ${ }^{*} \boldsymbol{T}$ the "upstream transition $»$ respectively located upstream and downstream of the arc to open;

3. Define $T i$ and $P P i$, respectively the first transition and the last place inserted in the GE;

4. Build the arc connecting the downstream place to the transition $\mathrm{Ti}\left(\boldsymbol{P}^{*} \rightarrow \boldsymbol{T i}\right)$;

5. Build the arc connecting the upstream transition in place PPI $\left(\boldsymbol{P P i} \rightarrow{ }^{*} \boldsymbol{T}\right)$;

6. Insert in the initial impact matrix, the rows and the columns pertaining respectively to the added places and transitions;

7. Set to 0 the cell $(P * * \boldsymbol{T})$;

8. Set to -1 the cells $\left(\boldsymbol{P}^{*}, * \boldsymbol{T} \boldsymbol{i}\right)$ and $(\boldsymbol{P P i}, * \boldsymbol{T})$;

9. Fill in the rest of the added cells while maintaining the rest of the original matrix.

Table 3. Impact Matrix resulting from a meeting of end panel: deletion of places, transitions and sub networks (in yellow)

\begin{tabular}{|c|c|c|c|c|c|c|c|c|c|c|c|c|c|c|c|c|c|c|c|c|c|c|c|}
\hline & $\mathrm{T} 1$ & TT1 & $\mathrm{Tt} 1$ & T2 & TT2 & $\mathrm{T} \mathrm{t} 2$ & $\mathrm{~T} 3$ & T4 & T5 & T6 & $\mathrm{T} 7$ & T8 & T9 & T10 & T11 & $\mathrm{T} 12$ & $\mathrm{~T} 13$ & T14 & $\mathrm{T} 15$ & $\mathrm{~T} 16$ & T17 & T18 & T19 \\
\hline P1 & -1 & 0 & 0 & 0 & 0 & 0 & 0 & 1 & 0 & 0 & 0 & 0 & 0 & 0 & 0 & 0 & 0 & 0 & 0 & 0 & 0 & 0 & 0 \\
\hline$P \Rightarrow \mathrm{P} 2$ & 1 & 0 & 0 & -1 & 0 & 0 & 0 & 0 & 0 & 0 & 0 & 0 & 0 & 0 & 0 & 0 & 0 & 0 & 0 & 0 & 0 & 0 & 0 \\
\hline P3 & 0 & 0 & 0 & 1 & 0 & 0 & -1 & 0 & 0 & 0 & 0 & 0 & 0 & 0 & 0 & 0 & 0 & 0 & 0 & 0 & 0 & 0 & 0 \\
\hline P4 & 0 & 0 & 0 & 0 & 0 & 0 & 1 & -1 & 0 & 0 & 0 & 0 & 0 & 0 & 0 & 0 & 0 & 0 & 0 & 0 & 0 & 0 & 0 \\
\hline P5 & 1 & -1 & 0 & 0 & 0 & 0 & 0 & 0 & 0 & 0 & 0 & 0 & 0 & 0 & 0 & 0 & 0 & 0 & 0 & 0 & 0 & 0 & 0 \\
\hline P6 & 0 & 0 & 0 & 1 & -1 & 0 & 0 & 0 & 0 & 0 & 0 & 0 & 0 & 0 & 0 & 0 & 0 & 0 & 0 & 0 & 0 & 0 & 0 \\
\hline P7 & 1 & -1 & 0 & 0 & 0 & 0 & 0 & 0 & 0 & 0 & 0 & 0 & 0 & 0 & 0 & 0 & 0 & 0 & 0 & 0 & 0 & 0 & 0 \\
\hline P8 & 0 & 0 & 0 & 1 & -1 & 0 & 0 & 0 & 0 & 0 & 0 & 0 & 0 & 0 & 0 & 0 & 0 & 0 & 0 & 0 & 0 & 0 & 0 \\
\hline P9 & 0 & 0 & 0 & 0 & 0 & 0 & 0 & 0 & 1 & 0 & 0 & 0 & 0 & 0 & -1 & 0 & 0 & 0 & 0 & 0 & 0 & 0 & 0 \\
\hline P10 & 0 & 0 & 0 & 0 & 0 & 0 & 0 & 0 & 0 & 1 & 0 & 0 & 0 & 0 & 0 & -1 & 0 & 0 & 0 & 0 & 0 & 0 & 0 \\
\hline P11 & 0 & 0 & 0 & 0 & 0 & 0 & 0 & 0 & 0 & 0 & 1 & 0 & -1 & 0 & 0 & 0 & 0 & 0 & 0 & 0 & 0 & 0 & 0 \\
\hline $\mathrm{P} 12$ & 0 & 0 & 0 & 0 & 0 & 0 & 0 & 0 & 0 & 0 & 0 & 1 & 0 & -1 & 0 & 0 & 0 & 0 & 0 & 0 & 0 & 0 & 0 \\
\hline P13 & 0 & 0 & 0 & 0 & 0 & 0 & 0 & 0 & 0 & 0 & -1 & 0 & 1 & 0 & 0 & 0 & 0 & 0 & 0 & 0 & 0 & 0 & 0 \\
\hline P14 & 0 & 0 & 0 & 0 & 0 & 0 & 0 & 0 & 0 & 0 & 0 & -1 & 0 & 1 & 0 & 0 & 0 & 0 & 0 & 0 & 0 & 0 & 0 \\
\hline P15 & 0 & 0 & 0 & 0 & 0 & 0 & 0 & 0 & 0 & 0 & 0 & 0 & 0 & 0 & -1 & -1 & 0 & 0 & 1 & 1 & 0 & 0 & 0 \\
\hline P16 & 0 & 0 & 0 & 0 & 0 & 0 & 0 & 0 & 0 & 0 & 0 & 0 & 0 & 0 & 1 & 1 & -1 & -1 & 0 & 0 & 0 & 0 & 0 \\
\hline P17 & 0 & 0 & 0 & 0 & 0 & 0 & 0 & 0 & 0 & 0 & 0 & 0 & 0 & 0 & 0 & 0 & 1 & 0 & -1 & 0 & 0 & 0 & 0 \\
\hline P18 & 0 & 0 & 0 & 0 & 0 & 0 & 0 & 0 & 0 & 0 & 0 & 0 & 0 & 0 & 0 & 0 & 0 & 1 & 0 & -1 & 0 & 0 & 0 \\
\hline P19 & 0 & 0 & 0 & 0 & 0 & 0 & 0 & 0 & 0 & 0 & 0 & 0 & 0 & 0 & 0 & 0 & 0 & 0 & 2 & 1 & -1 & 0 & 0 \\
\hline P20 & 0 & 0 & 0 & 0 & 0 & 0 & 0 & 0 & 0 & 0 & 0 & 0 & 0 & 0 & 0 & 0 & 0 & 0 & 0 & 0 & 0 & -1 & 1 \\
\hline $\mathrm{P} 21$ & 0 & 0 & 0 & 0 & 0 & 0 & 0 & 0 & 0 & 0 & 0 & 0 & 0 & 0 & 0 & 0 & 0 & 0 & 0 & 0 & 0 & 1 & -1 \\
\hline PT1 & 0 & -1 & -1 & 0 & 0 & 0 & 0 & 1 & 0 & 0 & 0 & 0 & 0 & 0 & 0 & 0 & 0 & 0 & 0 & 0 & 0 & 0 & 0 \\
\hline PT2 & 0 & 0 & 0 & 0 & -1 & -1 & 1 & 0 & 0 & 0 & 0 & 0 & 0 & 0 & 0 & 0 & 0 & 0 & 0 & 0 & 0 & 0 & 0 \\
\hline
\end{tabular}




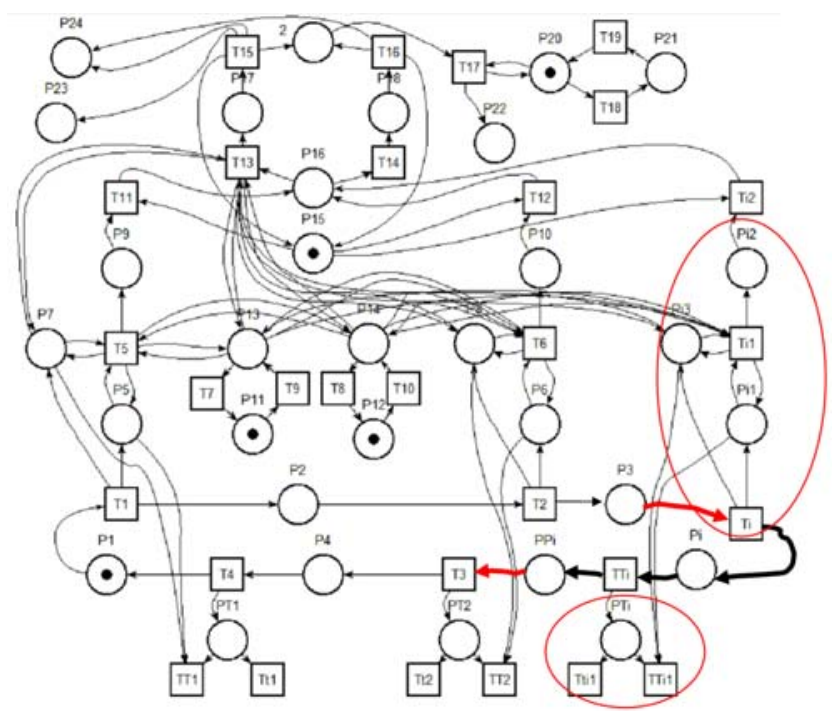

Figure 7. Modelling of the encounter of a 3rd signalling panel

Let's apply the algorithm to the one pair of the panel's model (Fig. 6) and its impact matrix (Table 3):

1. Open the arc $\mathrm{P} 2 \rightarrow \mathrm{T} 4$;

2. Set $\mathrm{P} 2=\boldsymbol{P}^{*}, \mathrm{~T} 4={ }^{*} \boldsymbol{T} ;$

3. Define $\mathrm{Ti}=\mathrm{T} 2$ and $\mathrm{PPi}=\mathrm{P} 4$;

4. Build the arc $\mathrm{P} 2 \rightarrow \mathrm{T} 2$;

5. Build the arc $\mathrm{P} 4 \rightarrow \mathrm{T} 4$;

6. Insert in the original matrix the lines P3, P4, P6, P8, P10, PT2 and the columns T2, TT2, Tt2, T3, T6, T12;

7. Set to 0 the cell $(\mathrm{P} 2, \mathrm{~T} 4)$;

8. Set to -1 the cells $(\mathrm{P} 2, \mathrm{~T} 2)$ and $(\mathrm{P} 4, \mathrm{~T} 4)$;

9. Fill in the remaining added cells, while maintaining the rest of the original matrix.

Table 4. Impact Matrix: effect of the encounter of $\mathrm{a}^{\text {th }}$ (here $3^{\text {th }}$ ) sign panel (in yellow the added rows and columns)

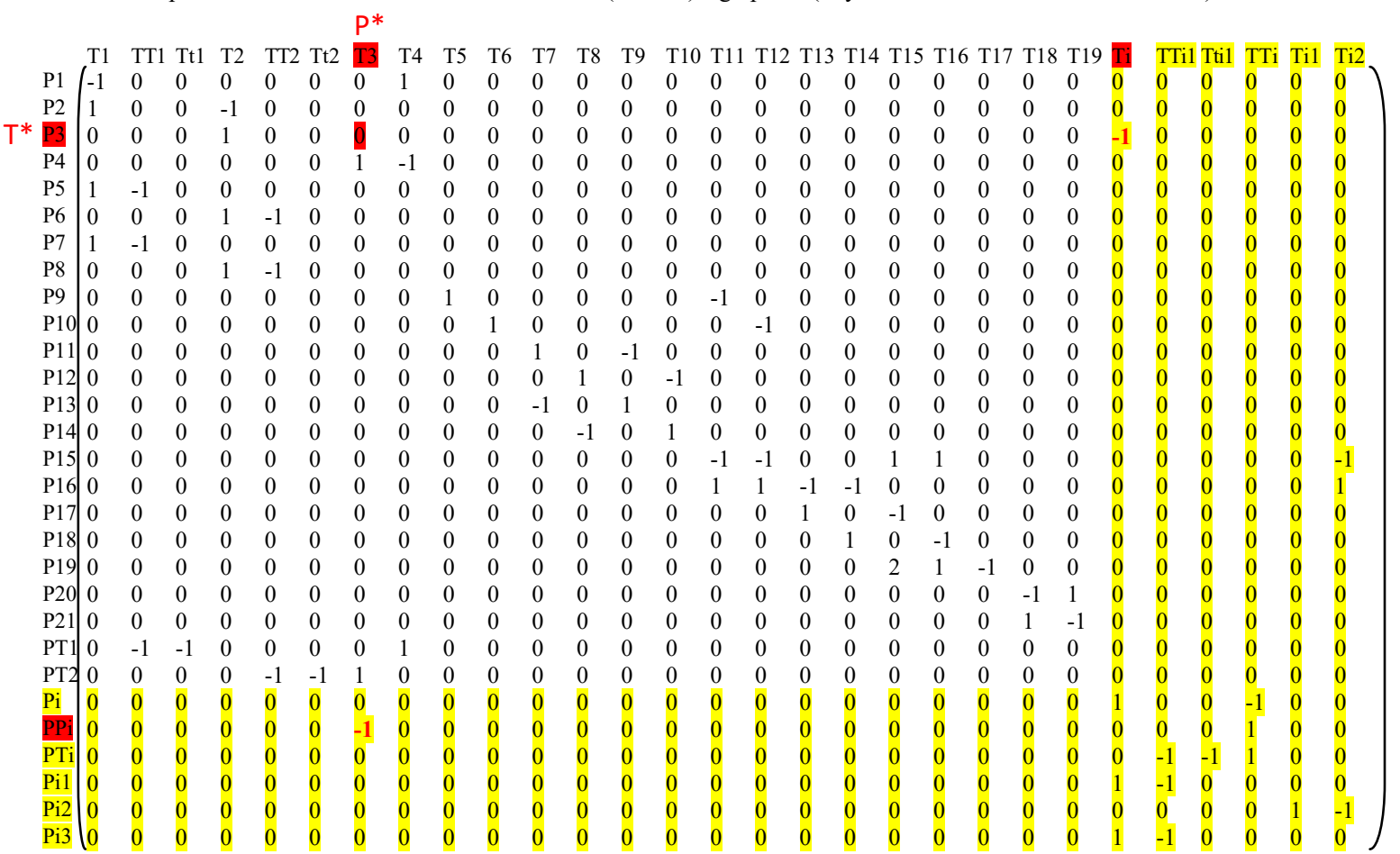


Let's apply the algorithm to that initial model (Fig. 2) and its impact matrix:

1. Open the $\operatorname{arc} \mathrm{P} 3 \rightarrow \mathrm{T} 3$;

2. Define P3 $=\boldsymbol{P}^{*}, \mathrm{~T} 3={ }^{*} \boldsymbol{T}$;

3. Define Ti and PPi;

4. Build the arc $\mathrm{P} 3 \rightarrow \mathrm{Ti}$;

5. Build the arc $\mathrm{PPi} \rightarrow \mathrm{T} 3$;

6. Insert in the original matrix, the lines Pi, PPi, PTi, Pi1, Pi2, Pi3 and the columns TTi1, Tti1, TTi, Ti1, Ti2;

7. Set to 0 the cell (P3, T3);

8. Set to $\mathbf{- 1}$ the cells $(\mathrm{P} 3, \mathrm{Ti})$ and (PPi, T3);

9. Filling the remaining added cells, while maintaining the rest of the original matrix.

Found well our Petri nets and its original matrix (Fig. 7) and its resulting impact matrix (Table 4).

\section{Conclusion}

We have studied the modelling with the Petri nets and then we have proposed a model for our system. The step-by-step and random simulation of this model with the tool TINA shows that the model meets the requirements of the specification. The analysis of the relevant properties of the model by the linear algebra shows that the only transitions that are blocking the network regardless of the initial marking are those of end panels (TT1, TT2 TTi1, etc.). They behave as switches which activate the process on the road section and disable it at the end of the said section. In addition, we propose two algorithms for the analysis of the model which guarantee that the latter retains its properties of absence of blocking and boundedness during its incremental evolution. This has enabled us to validate the model.

\section{References}

1. Atif, K. (1993) Modelling parallelism and synchronization. Thesis to obtain the degree of Doctor of the NPIG, Grenoble, France.

2. Bastide, R. (2000) Behavioural specification by Petri nets: Application to systems distributed objects and interactive systems. Habilitation to supervise researches of the University of Toulouse1, specialty Informatics, $74 \mathrm{p}$.

3. Belval. Road safety. [Online]. Available from www.westatlanticconsulting.com, accessed November 29, 2015.

4. Bennis, N. (2014) Petri Nets: Theory, Properties and Applications. [Online]. Available from website: http://www.specialautom.net, accessed December 08, 2015.

5. Brenac, T. (1997) Sequence analysis of road accident: How to put it into practice in diagnostics of road safety, tool and method. Research report INRETS, $\mathrm{n}^{\circ} 3,79 \mathrm{p}$.

6. Brenac, T. and Fleury, D. (1999) The concept of scenario type of traffic accident and its applications. Research Transport Security. 63, 63-76.

7. Cassandras, C.G. and Lafortune, S. (2008) Introduction to Discrete Event Systems. Second Edition. New York : Springer Science.

8. Chu, F. (1995) Conception of Production Systems with Petri Nets: Incremental verification of qualitative properties: $\mathrm{PhD}$ thesis in Automation, defence in Metz-France.

9. Fanga, C.Y., Fuhb, C.S., Yena, P.S., Cherngc, S. and Chend, S.W. (2004) An automatic road sign recognition system based on a computational model of human recognition processing. Computer Vision and Image Understanding, 96 (2004), 237-268.

10. Fernandes, P.H.L. (1998) Numerical methods for the solution of Markov systems with large state space. Thesis to obtain the degree of Doctor of the NPIG, Grenoble, France.

11. Hamdi Hocine. (2003) Automation Logic, Modelling and Control, Primer on Petri Nets. Constantine: Editions of the University of Mentouri.

12. International Federation of Red Cross and Red Crescent Societies; Global Road Safety Partnership. (2007) Road Safety Practical Guide. ISBN: 978-2-940395-02-6.

13. International Traffic Safety Data Group (2012) Road Safety. Annual Report 2011-0ECD/ITF, 7-12.

14. Kombe, T. (2011) Modeling of faults Propagation in production systems. PhD thesis defense on June 30, 2011, Ampere laboratory of National Institute of Applied Sciences of Lyon in France.

15. Leclercq, J.P. (2012) Courses of Graph Theory and Petri Nets. (IHDC-B321). 
16. Organization for Economic Cooperation and Development. (1988) Road accidents, site investigations. Paris, $113 \mathrm{p}$.

17. World Health Organization. (2009) Global Status Report on Road Safety. Time for Action, Geneva, $301 \mathrm{p}$.

18. Young, W., Sobhani, A., Lenne, M.G., and Sarvi, M. (2014) Simulation of safety: A review of the state of art in road sefety simulation modelling, Accident Analysis \& Prevention, volume 66, 2014, p 89-103.

19. Zogo Tsala, S.A., Makomra, V. and Ayina Ohandja, L.M. (2015) Typology of road accidents related to the default of signaling: A case study of the Yaounde-Douala Highway, southern Cameroon. Journal of Transportation Technologies, 5, 122-133. 